PREFACE

\title{
Primary Care Practice-Based Research Comes of Age in the United States
}

\author{
David Lanier, MD \\ Center for Primary Care, Prevention, and Clinical Partnerships, Agency for Healthcare Research and Quality, Rockville, Md
}

Ann Fam Med 2005;3(Suppl 1):S2-S4. DOI: 10.1370/afm.338.

$\mathrm{T}$ The enormous potential of practice-based research networks (PBRNs) to expand the knowledge base of primary care and to integrate research into practice (and practice into research) was recognized in the United States more than 25 years ago. Beginning with the development of early regional PBRNs in the late $1970 s^{1,2}$ the feasibility of conducting research in networks of primary care practices was quickly established. Throughout the 1980s and early 1990s, a steady stream of research reports from local, regional, and national practice networks then began appearing in the medical literature as the enterprise slowly grew and spread. Despite these successes, however, it was quite apparent that PBRNs as a group continued to face a number of challenges. Chief among these challenges was the absence of any systematic support for the growth and maturation of PBRNs. In its 1996 report on primary care, the Institute of Medicine viewed PBRNs as "a significant underpinning for studies in primary care" but noted that they were underfunded. One of the report's recommendations was that "the Department of Health and Human Services provide adequate and stable ... support to practice-based primary care research networks."3

A few years later, the Agency for Healthcare Research and Quality (AHRQ) responded by releasing the first of a series of grant solicitations specifically targeting primary care PBRNs. From 2000 to 2004, AHRQ has made awards to primary care PBRNs totaling more than $\$ 8$ million. Although the individual

Conflict of interest: none reported

\section{CORRESPONDING AUTHOR}

David Lanier, MD

Center for Primary Care, Prevention, and Clinical Partnerships Agency for Healthcare Research and Quality

John M. Eisenberg Building

540 Gaither Rd

Rockville, MD 20850

David.Lanier@ahrq.gov awards have been modest, the number and diversity of networks applying for these funds have been remarkable. The 45 networks that have to date received AHRQ funding for infrastructure support and pilot projects include among their members more than 10,000 primary care clinicians who care for more than 10 million Americans. In addition, in 2002 the agency invested in the establishment (through a contract with Indiana University and the National Opinion Research Center) of a PBRN Resource Center that has been given the task of assessing the developmental needs of AHRQ-funded networks and helping to meet these needs through educational, technical, and consultative services.

During the past decade, the number and diversity of identifiable primary care networks in the United States have increased dramatically. A brief communication published in 1994 reported that 28 primary care PBRNs were active at that time in North America. ${ }^{4}$ Most of these networks consisted predominantly, if not entirely, of family physician practices. By 2004, a national survey (conducted by the PBRN Resource Center) identified 111 networks in the United States that met certain established criteria for being an active primary care PBRN. ${ }^{5}$ The survey revealed that multiple types of practitioners, including pediatricians, general internists, and advanced practice nurses (in addition to family physicians), are represented in these networks, which are headquartered in 44 states. The data also indicated that a large number of these networks first emerged after 2000, when the seed money and other support offered to PBRNs by AHRQ first became available.

The clear challenge now facing the agency is how to continue supporting the growth and maturation of primary care PBRNs across the country in an era of projected federal budgetary limitations but with many more networks potentially needing our support. One approach has been to forge partnerships with other funding sources in both the private and the federal 
sectors. A major AHRQ collaborator in supporting primary care PBRN efforts has been the Robert Wood Johnson Foundation, which in 2003 began investing $\$ 5$ million in "innovation grants" awarded to selected PBRNs to identify and test interventions aimed at changing 2 or more modifiable risky health behaviors within the context of primary care practice. ${ }^{6}$ In addition, several initiatives aimed at promoting the development of clinical research networks and included in the new National Institutes of Health Roadmap (http:// nihroadmap.nih.gov/) have provided funding for building infrastructure within a few primary care PBRNs. Although several institutes of the National Institutes of Health have supported individual PBRN projects, the National Cancer Institute has been AHRQ's major collaborator in funding network-based projects. For example, AHRQ and the National Cancer Institute are currently cosponsoring program announcements supporting investigations of PBRNs into methods of screening for colorectal cancer and methods of translating research into primary care practice.

Another way in which AHRQ hopes to continue promoting the growth and maturation of PBRNs is through the provision of regularly updated educational information to both emerging and established PBRNs on optimal methods of addressing a variety of new and continuing challenges that face networks. The articles included in this AHRQ-supported supplement to the Annals of Family Medicine were written with this educational goal in mind. To identify the most pertinent topics and content to be covered in the supplement, as well as the preferred author(s) for each article, AHRQ asked the supplement editor, Dr Paul Nutting, to work with an advisory panel composed of a multidisciplinary group of individuals who are recognized leaders in network activities.* In addition, the PBRN Resource Center provided the editor with useful summary information derived from its assessment of the specific educational needs of networks it surveyed across the country.

The articles presented in this supplement address a wide range of topics and issues pertinent to networks at all stages of development. The article by Green et $\mathrm{al}^{7}$ on optimizing network infrastructure should be particularly useful for newly established or emerging PBRNs. It goes beyond the question of funding sources to describe the basic elements required by most or all PBRNs and how those requirements typically depend on the specific research mission of the network. Since releasing the first PBRN grant solicitation, AHRQ has been keenly interested in the development by PBRNs of systems for electronic data collection and aggre-

*The advisory panel included Andrew Bindman, MD, MPH; Walter Calmbach, MD; Benjamin Crabtree, PhD; Margaret Grey, DrPH, CPNP; John Hickner, MD, MS; Wilson Pace, MD; Richard Wasserman, MD, MPH; and Barbara Yawn, MD, MS. gation. As Pace and Staton ${ }^{8}$ point out, however, the benefits of these systems (such as improved data entry and integrity and easier data transfer) must be weighed against both their costs and the potential burden they place on research participants.

Because the relationships between primary care clinicians and their patients often span years or decades, PBRN researchers often have the opportunity to consider not only data from a single encounter, but also information collected longitudinally that spans entire episodes of care and describes the ongoing management of chronic health problems and the provision of health maintenance. The article by van $\mathrm{Weel}^{9}$ describes the benefits and the challenges of research that focuses on longitudinally collected data.

Even as data are collected, however, PBRN researchers need to be ever mindful of the potential effects of the Health Insurance Portability and Accountability Act (HIPAA) on the handling of patients' protected health information. The article by Pace et $\mathrm{al}^{10}$ explores the ways in which HIPAA can affect research conducted in PBRNs. Closely related to concerns about HIPAA are important issues surrounding the review and approval of PBRN projects by institutional review boards (IRBs). Although the actual risks incurred by patients through participation in PBRN research are typically minimal, the process of seeking IRB approval can often be complicated and protracted for networks, which are sometimes required to have protocols reviewed by multiple IRBs. The article by Wolf et al ${ }^{11}$ offers suggestions and options for networks to consider as they work with their local IRBs.

Finally, the article by Mold and Peterson ${ }^{12}$ on networks as quality improvement and learning collaboratives appears to challenge the traditional definition of PBRNs as research laboratories. AHRQ's work with PBRNs, however, has helped us recognize the potential of primary care networks to expand their purposes beyond traditional research to developing places of learning for clinicians. Equal to the goal of publishing research results should be the goal of nurturing an evidence-based culture in primary care. As Mold and Peterson ${ }^{12}$ indicate, engaging network practitioners in reflective inquiries can lead to practice improvement as well as to new researchable questions for the network.

The articles included in this supplement provide important information for those wanting to realize the full potential of network activities. AHRQ's intention is to make this information widely available to all primary care PBRNs in the United States and to expand and update it periodically over the years to come. Through these and other efforts, we hope to contribute in a major way to the agency's long-term objectives for PBRNs: to improve the capacity of practice networks to 
conduct high-quality research that expands the primary care knowledge base, and to improve the primary care of patients in the United States by ensuring that new knowledge is incorporated into actual practice.

To read or post commentaries in response to this article, see it online at http://www.annfammed.org/cgi/content/full/3/Suppl_1/S2.

\section{References}

1. Green LA, Simmons RL, Reed FM, Warren PS, Morrison JD. A family medicine information system: the beginning of a network for practicing and residency family physicians. J Fam Pract. 1978;7:567-576.

2. Nelson EC, Kirk JW, Bise B, et al. The cooperative information project: Part 1: a sentinel practice network for service and research in primary care. J Fam Pract. 1981;13:641-649.

3. Institute of Medicine. Primary Care: America's Health in a New Era. Washington, DC: National Academy Press; 1996.

4. Niebauer L, Nutting PA. Primary care practice-based research networks active in North America. J Fam Pract. 1994;38:425-426.
5. Tierney WM, Hudson BL, Oppenheimer CC, et al. A national survey of primary care practice-based research networks. J Gen Intern Med. 2004;19(Suppl 1):111.

6. The Robert Wood Johnson Foundation. Prescription for health. Web site. Available at: http://www.prescriptionforhealth.org.

7. Green LA, White LL, Barry HC, Nease DE Jr, Hudson BL. Infrastructure requirements for practice-based research networks. Ann Fam Med. 2005;3(Suppl 1):S5-S11.

8. Pace WD, Staton EW. Electronic data collection options for practicebased research networks. Ann Fam Med. 2005;3(Suppl 1):S21-S29.

9. van Weel C. Longitudinal research and data collection in primary care. Ann Fam Med. 2005;3(Suppl 1):S46-S51.

10. Pace WD, Staton EW, Holcomb S. Practice-based research network studies in the age of HIPAA. Ann Fam Med. 2005;3(Suppl 1):S38-S45.

11. Wolf LE, Walden JF, Lo B. Human subjects issues and IRB review in practice-based research. Ann Fam Med. 2005;3(Suppl 1):S30-S37.

12. Mold JW, Peterson KA. Primary care practice-based research networks: working at the interface between research and quality improvement. Ann Fam Med. 2005;3(Suppl 1):S12-S20. 